La

Révolution

française

\section{La Révolution française}

Cahiers de l'Institut d'histoire de la Révolution française

$9 \mid 2015$

Citoyenneté, république, démocratie dans la France de la Révolution

\title{
Emmanuel de WARESQUIEL, Fouché, les silences de la pieuvre
}

Paris, Fayard, 2014

Jeanne-Laure le Quang

\section{OpenEdition}

\section{Journals}

Édition électronique

URL : http://journals.openedition.org/lrf/1427

DOI : $10.4000 /$ Irf. 1427

ISSN : 2105-2557

Éditeur

IHMC - Institut d'histoire moderne et contemporaine (UMR 8066)

Référence électronique

Jeanne-Laure le Quang, «Emmanuel de WaresquieL, Fouché, les silences de la pieuvre », La Révolution

française [En ligne], 9 | 2015, mis en ligne le 16 novembre 2015, consulté le 24 septembre 2020. URL http://journals.openedition.org//rf/1427 ; DOI : https://doi.org/10.4000/Irf.1427

Ce document a été généré automatiquement le 24 septembre 2020.

(c) La Révolution française 


\section{Emmanuel de WARESQUIEL, Fouché, les silences de la pieuvre}

Paris, Fayard, 2014

Jeanne-Laure le Quang

\section{RÉFÉRENCE}

Emmanuel de Waresquiel, Fouché, les silences de la pieuvre, Paris, Fayard, 2014, 831 pages 
1 «La pieuvre, en chasse ou au guet, se dérobe; elle se rapetisse, elle se condense ; elle se réduit à sa plus simple expression. Elle se confond avec la pénombre. Elle a l'air d'un pli de la vague. Elle ressemble à tout, excepté à quelque chose de vivant. Elle n'a pas d'os, elle n'a pas de sang, elle n'a pas de chair. C'est une peau. De la glu pétrie de haine. Toute la bête est froide. [...] Presque toujours, quand on la voit, on est pris. " C'est " un chef d'œuvre, si l'épouvante est un but ${ }^{1}$. » C'est en relisant cet extrait des Travailleurs de la mer de Victor Hugo qu'Emmanuel de Waresquiel a trouvé le sous-titre de sa biographie de Joseph Fouché: «la description terrifiante » qu'Hugo fait de la pieuvre «m'a fait irrésistiblement penser

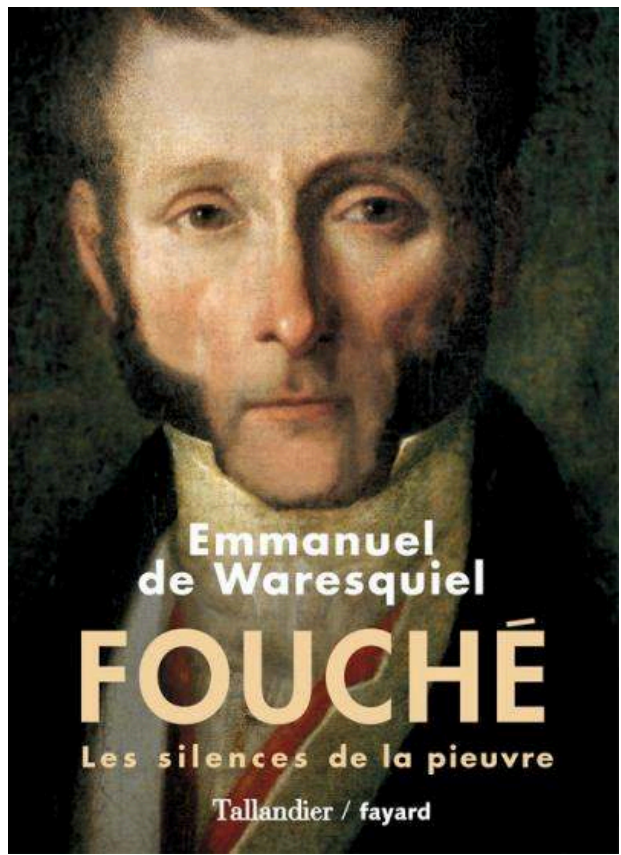
à Fouché », dit-il ${ }^{2}$. Nul n'est besoin de rappeler, de fait, le pouvoir de fascination qui entoure Fouché, gangue légendaire que l'auteur évoque et qu'il entend déconstruire. Le sous-titre évoque à la fois le mythe du ministre de la police omniscient et omnipotent, étendant ses tentacules dans toutes les ramifications de la société, et les «silences" de l'homme insaisissable, fuyant, impassible, difficile à mettre à jour derrière le masque.

De fait, à travers cette biographie monumentale (42 chapitres, 831 pages), l'ambition d'Emmanuel de Waresquiel est d'élaborer une nouvelle synthèse scientifique exhaustive. La dernière somme consacrée à ce personnage clé des périodes révolutionnaire et impériale, comme des débuts de la Restauration, date en effet de 1901. C'est la thèse écrite par Louis Madelin'3 , tenant de l'histoire positiviste, qui a le premier cherché à briser la légende forgée au XIX ${ }^{\mathrm{e}}$ siècle, abondamment relayée par ses plus grands auteurs, de Balzac à Hugo, pour y substituer un travail scientifique, dépassionné, appuyé sur un travail d'archives colossal. Depuis cette thèse pionnière et magistrale, des biographies consacrées à Fouché ont été publiées régulièrement, mais n'ont que peu renouvelé l'approche, se contentant de reprendre souvent une suite d'anecdotes savoureuses confinant encore au mythe ${ }^{4}$. D'autres ont été délibérément orientées : Stefan Zweig a livré ainsi une «étude psychologique » de Fouché à l'usage de ses contemporains, dans un contexte de montée des totalitarismes d'entre-deuxguerres $^{5}$, qui a néanmoins servi de source d'inspiration indéniable à la présente biographie pour certains passages où l'auteur donne à son récit une intensité dramatique $^{6}$. Enfin, nombre de biographies se concentrent sur un aspect seulement du personnage - c'est le cas de Jean Savant ${ }^{7}$ qui étudie Fouché à travers ses relations à Napoléon, ou de Jean Tulard qui a choisi de montrer un Fouché «inventeur» de la police moderne ${ }^{8}$.

3 Emmanuel de Waresquiel renoue donc avec un travail colossal dans la veine de Madelin, dont il assume l'apport décisif, cherchant à englober tous les aspects de la vie de l'homme, les sphères publiques comme privées, de la petite enfance aux dernières années d'exil. Il faut saluer l'effort d'équilibre de l'ouvrage entre les périodes 
révolutionnaire, consulaire et impériale et celle de la Restauration, mais aussi l'intérêt porté à l'enfance et la formation de Fouché, que l'auteur a bien plus fouillées que ses nombreux prédécesseurs. De Waresquiel est ainsi le premier historien à revenir en détail sur la famille de Fouché ${ }^{9}$, en situant l'ascension sociale de celui-ci dans une histoire familiale élargie. Alors que ses prédécesseurs se bornaient à mentionner que son père était capitaine de navire et possédait des terres à Saint Domingue, l'auteur de la présente biographie révèle qu'il était négrier et planteur, et a donc occupé une place - bien que modeste - dans la traite négrière dont Nantes constitue une plaque tournante.

4 Le souci d'exhaustivité de son travail se nourrit de la quantité impressionnante d'archives et de sources convoquées par l'auteur, au cours des cinq années de travail qu'a nécessité l'ouvrage. E. de Waresquiel a en effet repris les archives dépouillées pour la première fois par Madelin, mais aussi celles utilisées par les biographes successifs notamment les Mémoires de Fouché lui-même, que Madelin avait laissés de côté au moment de sa thèse parce qu'on en contestait l'authenticité, depuis en partie attestée -. Cependant, un apport indéniable de son travail est d'envisager un grand nombre d'archives restées encore inédites, à commencer par les archives privées de la famille Fouché, conservées par les descendants du notaire du fils de Fouché, soit huit cartons de brochures et lettres diverses, qui comprennent notamment «tous les comptes secrets de la police depuis la fin du Directoire jusqu'en 1806 » et « une bonne partie de la correspondance familiale de Fouché, à sa femme, à ses enfants ${ }^{10}$. » Les recherches de l'auteur l'ont également porté à découvrir d'autres fonds d'archives inédits, notamment aux archives de l'Oratoire, mais aussi des archives familiales en Suède (où deux fils de Fouché se sont installés), et faire des trouvailles intéressantes dans les catalogues de ventes publiques ou de librairies. Enfin, l'auteur utilise, beaucoup plus que ses prédécesseurs, le fourmillement de Mémoires des acteurs contemporains publiés sous la Restauration, notamment deux Mémoires inédits auxquels il puise énormément, ceux de Maurice Gaillard ${ }^{11}$, et ceux de Villiers du Terrage ${ }^{12}$.

5 La grande richesse des sources utilisées est mise par l'auteur au service de la grande ambition de l'ouvrage: renouveler l'approche de Joseph Fouché en se penchant sur l'homme lui-même, sa personnalité, son caractère et ses idées. E. de Waresquiel déplore dès l'avant-propos la focalisation de ses prédécesseurs sur le personnage politique, le masque, disant notamment de la thèse de Madelin qu' " on y distingue mal [...] les replis du cœur et de l'âme ${ }^{13}$.» En clair, le but affirmé est de sortir des "mythes » et de « revenir à l'homme, à ce qu'il a été, à ce qu'il a voulu, à ce qu'il a pensé. Là, les choses sont autrement plus subtiles. On est toujours avec lui du côté de l'ambivalence et des contradictions ${ }^{14}$." L'accès privilégié de l'auteur aux archives privées de la famille Fouché lui ont permis d'y «surprend[re] un autre homme, un cœur tendre, un grand sensible si différent de celui que l'on connaît, le politique, celui qui prépare ses coups de loin, enfermé dans son mutisme et sa patience, dissimulé sous le masque impassible des grands cyniques ${ }^{15}$. " L'auteur s'est donc attaché à montrer, bien plus que Louis Madelin ${ }^{16}$, l'homme privé. Emmanuel de Waresquiel souligne d'abord la grande proximité affective du Fouché professeur avec ses élèves de l'Oratoire ${ }^{17}$, puis décrit Fouché au sein de sa famille, en homme sensible, vouant un amour intense à ses deux femmes successives et à ses enfants : "s'il y a de la tendresse en lui, et il y en a à revendre, elle va tout entière à ses enfants qu'il gâte trop et élève dans un joyeux laisser-aller qui détonne avec l'image parfaitement insensible que l'on a habituellement de l'homme public ${ }^{18}$.» Les moments d'exil ou de disgrâce de Fouché sont, l'auteur le 
souligne, des périodes de vie en autarcie, où Fouché goûte aux plaisirs tranquilles de la vie de famille. Cette nouvelle biographie s'attache enfin aux dernières années de la vie de Fouché, loin de la France, "celles d'une longue et lente introspection », où l'homme « souffre ${ }^{19}$ ", comme en témoignent ses innombrables lettres à ses proches en France, comme Delphine de Custine, dans lesquelles l'auteur décèle « beaucoup de tendresse et de sensibilitée ${ }^{20}$. " De Waresquiel brosse donc un personnage débarrassé de sa légende noire, loin de la condamnation de l'homme sanguinaire et amoral qui a longtemps prévalue.

$6 \quad$ Il faut par ailleurs souligner le souci de l'auteur de se démarquer de ses prédécesseurs. À plusieurs reprises, Emmanuel de Waresquiel propose des interprétations différentes d'épisodes particuliers de la vie de Fouché ou de la scène politique. L'auteur bat d'abord en brèche l'idée de ses précédents biographes de la modération de Fouché au début de la Révolution, quand il est à la tête du collège de Nantes. Alors qu'on avait jusqu'alors dit qu'il n'était qu'un "pur opportuniste», ne se durcissant que quand la Révolution elle-même se durcit avec l'abolition de la royauté en septembre 1792, E. de Waresquiel insiste sur la théorie politique déjà cohérente de Fouché - notamment par l'étude de ses discours de 1791 à la société des Capucins, où on «trouve déjà les thèses du futur conventionnel et du terroriste en mission à Lyon» soit des "positions quasiment extrémistes »: primauté de la volonté générale, transparence du pouvoir et contrôle par les citoyens de leurs représentants, égalité, vision du roi comme obstacle ${ }^{21}$. De manière générale, E. de Waresquiel rejette le postulat de l'opportunisme d'un Fouché changeant de position au gré des circonstances, en y voyant au contraire du "pragmatisme", notamment au moment du vote sur la mort du roi, et dans les oscillations de Fouché entre Girondins et Montagnards - Fouché aurait fait délibérément feint d'être du côté des Girondins alors qu'il pencherait en réalité depuis son séjour à Nantes pour les Montagnards, avant de tomber le «masque » le 14 mars $793^{22}$-. De même, au moment du passage au Consulat à vie, Fouché aurait encouragé en sous-main, et non réprouvé celui-ci comme ses précédents biographes le pensaient. E. de Waresquiel assure même que Fouché, pour qui ce changement de régime ne va pas assez loin, voudrait mettre en place l'hérédité du pouvoir dès $1802^{23}$.

D'autre part, Emmanuel de Waresquiel accorde une importance nouvelle à la place du régicide dans la vie de Fouché. Il insiste à de multiples reprises sur ce poids qui pèse sur toute son existence. Pour l'auteur, c'est le fait d'avoir voté la mort du roi en 1793 qui expliquerait la disgrâce de Fouché alors ministre de la police de Napoléon en 1810. «Dans le contexte du mariage avec la petite-nièce de Marie-Antoinette», les " convenances" ne permettraient plus à Napoléon d'avoir dans son entourage des meurtriers de son grand-oncle par alliance ${ }^{24}$. De même, au moment de la première Restauration en 1814, c'est son statut de régicide qui expliquerait que Fouché est marginalisé et écarté du pouvoir, alors même qu'il apparaît comme « indispensable » et que tous lui demandent conseil, du favori du roi, le comte de Blacas, à Metternich ${ }^{25}$. Enfin, quand en juillet 1815, Fouché accepte le poste de ministre de la police de Louis XVIII, lors de la seconde Restauration, alors que Louis Madelin assène que « [...] la postérité doit se montrer sévère, sans injustice, pour l'homme sans scrupules et sans pudeur qui, ce jour-là, prostitua la Révolution aux pieds des Bourbons ${ }^{26} »$, la vision d'E. de Waresquiel est plus originale : « c'est surtout du côté de son passé qu'il faut chercher les raisons profonds de ce grand écart." "Avec le retour des Bourbons, son vote de janvier 1793 fait désormais de lui un paria en sursis. [...] Derrière l'armure protectrice de l'ambition et du pouvoir, sinon du cynisme, cet homme-là est désespérément seul et 
c'est bien ce face-à-face mortel avec lui-même qu'il redoute le plus. Il ne veut pas tomber pour avoir tué un roi. Son entrée dans le gouvernement de Louis XVIII est sa planche de salut, le serment qu'il vient de prêter au roi, son absolution ${ }^{27}$. »

On le voit, le style de l'auteur est vivant, dramatique, volontiers grandiloquent. Il est en effet central de souligner que tant le format - 42 chapitres aux titres romanesques que l'écriture font de l'ouvrage un livre accessible, ayant l'ambition de toucher un large lectorat, au-delà de la communauté scientifique. Et de fait, l'ouvrage a connu un succès de librairie et obtenu deux prix $^{28}$, saluant la vivacité d'un style qui plonge dans la littérature, puisant son inspiration dans un Balzac, un Hugo ou un Michelet, abondamment cités dans le texte afin d'accroître la puissance dramatique de certains récits, et ne ménageant pas les descriptions de l'apparence physique ou psychologique des personnages citées, ou des lieux traversés. Si la biographie est pour l'auteur un genre aux "allures interlopes", un "récit, avec ses enchaînements, ses apparences logiques, ses effets de surprise, sa vitesse et ses lenteurs ${ }^{29}$ ", et si on ne peut dénier au livre une réelle cohérence scientifique, on peut regretter que les effets de style soient parfois malvenus, et que la fascination que le personnage semble exercer sur l'auteur rende peu aisée la prise de distance nécessaire à l'exercice historique.

De fait, toute la première moitié de l'ouvrage (jusqu'au passage au Consulat) dresse un portrait psychologique de Fouché qui tend à l'essentialiser comme l'incarnation du secret, de l'ombre. Est décrite une enfance "marquée du sceau de la mort» (de ses frères et sœurs, de son père): " il y a là assez de manques et de ruptures pour développer certaines fragilités affectives, une instabilité qui le marquera pour la vie. Et puis la solitude de la mort incite naturellement au silence, à la dissimulation et au mutisme. De quoi vous forger un caractère ${ }^{30}$. « «Son tempérament naturellement porté à la dissimulation, son expérience révolutionnaire des conspirations vont faire de lui le grand maître du secret. [...] Avec lui, le secret est partout. [...] Voilà l'homme, tapi au centre de sa toile, caché dans l'écheveau de ses traces ${ }^{31}$." L'auteur a par ailleurs tendance à lire le passé à l'aune du futur, expliquant la «nature » secrète, dissimulatrice et machiavélique de celui qu'il nomme abondamment, de manière quelque peu malvenue, "le futur ministre de la police de Napoléon ", par son enfance et ses années à l'Oratoire.

10 Sa lecture des années révolutionnaires se fait avec la même grille d'analyse: "Après avoir été un temps adepte de la maison de verre [c'est-à-dire de la transparence], les conditions de l'exercice du pouvoir en révolution le feront très vite revenir à ce qu'il est fondamentalement, par tempérament et par caractère, l'homme des mécanismes de l'opacité et du secret ${ }^{32}$.» Au milieu de démonstrations scientifiques pourtant souvent approfondies et passionnantes, notamment la brillante description de l'ascension progressive de Fouché sous la Terreur, permise par son rôle de représentant en mission (à Nevers, Moulins et Lyon notamment), ou la manière dont, dans ces villes, Fouché organise une véritable régénération révolutionnaire ( un programme de révolution totale $\left.^{33} »\right)$, on peut regretter l'existence de généralisations confinant au mythe d'un Fouché tout-puissant, évoquant les subalternes de Fouché comme « ses créatures ${ }^{34}$ », ou sa personnalité naturellement machiavélique de "grand prédateur à sang froid ${ }^{35}$ ", ainsi que les nombreuses métaphores de la bestialité parsemant le texte, de l'araignée ${ }^{36}$ au mollusque ${ }^{37}$.

11 En définitive, par son style littéraire, peut-être mû par l'ambition grand public de l'ouvrage, et quelques raccourcis fâcheux, l'auteur semble contribuer à nouveau au 
mythe du personnage. Si le présent ouvrage extrait définitivement Fouché de sa légende noire, il pourrait paradoxalement constituer en lui-même - et pour ainsi dire malgré lui - une pierre de plus à la construction d'une autre légende, parallèle et entrecroisée, une légende d'un Fouché tout puissant et tirant toutes les ficelles.

Néanmoins, la deuxième moitié du livre, concernant les années 1799-1820, quitte en grande partie la dimension psychologique et apparait indéniablement plus novatrice sur le plan scientifique. Les historiens trouveront dans cette seconde moitié des apports réels à la connaissance de Fouché, qui acquiert une complexité encore inégalée.

Tout d'abord, la biographie s'attache à s'éloigner de la légende noire en insistant sur "l'esprit de modération ${ }^{38}$ » dont fait preuve Fouché, à de nombreuses reprises au cours de sa carrière politique. E. de Waresquiel met ainsi à jour dans un passage convaincant les conseils que Fouché adresse aux préfets, à partir de l'Empire. Des conseils de modération, et de discrétion dans la répression, afin de ne pas alimenter les mécontentements de la société impériale: «Les préfets doivent offrir à leurs administrés le spectacle d'un Empire apaisé, débarrassé des passions et de l'esprit de parti $^{39}$. » Il s'agit donc bien d'une modération encouragée avant tout par pragmatisme politique : avoir une action mesurée permet d'apaiser à la fois les tensions politiques en ralliant les anciens partis derrière l'Empereur, mais aussi les tensions populaires liées à la conscription en province ${ }^{40}$. De même, l'auteur exhume un texte méconnu écrit par Fouché ${ }^{41}$, parlant de la nécessité de conserver une petite part d'opposition contrôlée, qui contraste fortement avec l'image fantasmée d'un ministre enfermant à tour de bras. Pour l'auteur, cette modération de Fouché, perçue comme un "art de gouverner ${ }^{42}$ " serait en décalage avec le tournant autoritaire d'un Empereur qui ne supporte pas les opinions divergentes, refuse toute liberté de parole, mais n'empêcherait pas de mener une réelle répression, si celle-ci est discrète et sans vagues.

D'autre part, alors que ses prédécesseurs ne faisaient de Fouché qu'un opportuniste, l'auteur de la présente étude insiste au contraire sur la cohérence de ses idées, de sa théorie politique, à travers tous les régimes et «au-delà de ses revirements politiques $^{43}$." Cette cohérence réside dans son attachement aux acquis révolutionnaires, qu'il cherche toujours à sauvegarder à partir du Consulat, alors que la Révolution est peu à peu enterrée. L'auteur démontre de manière convaincante que, pendant les Cent-Jours, « au sein du Conseil de Napoléon, Fouché se montre inflexible sur la question des libertés ${ }^{44} "$, en faisant supprimer la censure, en empêchant la création de commissions militaires chargées de juger les "traîtres" dans les départements, en incitant vivement ses lieutenants de police à une action juste et respectueuse des libertés et des lois, et enfin, en poussant Napoléon à avoir une conduite modérée, faite de clémence et d'indulgence, le tout afin de préserver les acquis révolutionnaires. De même, à la chute des Cent-Jours en 1815, alors que Fouché, président du gouvernement, est, pour ses contemporains comme ses biographes, l'homme de "toutes les volte-face", donnant à chaque parti l'assurance qu'il les soutenait (Bourbons, libéraux, Jacobins, alliés...), Emmanuel de Waresquiel peint un homme qui ne sait pas vraiment quoi faire, mais veut avant tout garantir «la Révolution et ses acquis »: " négocier au plus serré les conditions d'accès au trône du candidat qui s'y présentera», et obtenir des alliés des "concessions constitutionnelles ${ }^{45}$.» Enfin, l'auteur restitue toute la complexité du jeu politique de Fouché, nommé ministre de la police de Louis XVIII en 1815, jeu politique qui consiste à

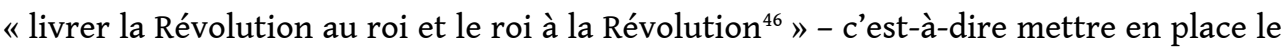


nouveau régime tout en sauvegardant les acquis de la Révolution. L'attachement profond de Fouché aux acquis révolutionnaires est ainsi un fil rouge nouveau, qui permet de relire sa vie et les différentes fonctions qu'il occupe, et qui pour lui importerait plus que le souverain lui-même.

Par ailleurs, ce portrait de la carrière politique de Fouché à partir du Consulat comporte des développements techniques novateurs. On peut d'abord soulever l'apport décisif permis par l'étude de la comptabilité des fonds secrets du ministère de la police ${ }^{47}$ à laquelle un historien a eu pour la première fois accès. Par l'analyse précise de ces dépenses secrètes et de leur évolution ${ }^{48}$, E. de Waresquiel apporte une pierre nouvelle à la connaissance de la police impériale. Il révèle l'augmentation de ces fonds secrets - issus du produit des maisons de jeu - au cours de la période (1799-1810), et le fait qu'ils représentent une somme supérieure au budget officiel de la police. Si l'analyse donne un aperçu beaucoup plus clair du personnel de la police secrète, elle met également à jour le fait que ces fonds servent non seulement au recrutement d'agents réguliers et de mouches dans tous les milieux de la société, mais également à des subsides versées, pêle-mêle, à des journaux français comme étrangers, aux théâtres parisiens, "à divers hommes de lettres à titre d'encouragement ", ou encore "à divers secours attribués sur ordre du ministre d'après une liste nominative. » Waresquiel en conclut très justement que "cela donne une idée du nombre de ses obligés et des leviers considérables qu'il pouvait avoir sur l'opinion ${ }^{49}$.» On aurait aimé néanmoins que ces tableaux comptables soient publiés par l'auteur en annexe de son ouvrage.

Dans la même optique, l'auteur met à jour avec justesse le souci de rationalisation et de statistique qu'a Fouché, et ce, précocement, dès le moment où il est représentant en mission à Lyon : Fouché a une vision «administrative et comptable » de la Terreur, qui s'incarne dans sa volonté d' "unifier, simplifier, rationaliser, centraliser», en demandant à ses subalternes des «tableaux, des listes, des formulaires, des inventaires qu'il multiplie de façon exponentielle sur tous les sujets et dans tous les domaines, hommes et $\operatorname{choses}^{50}$. » Ce souci de rationalisation s'amplifie à partir du moment où il est ministre de la police. Les archives fourmillent de demandes de Fouché aux autorités locales ou aux préfets de rapports réguliers, esquissant par exemple un " premier plan de «statistique personnelle et morale » en 1807 afin d'obtenir « un vaste tableau de l'Empire dans tous ses éléments constitutifs à tous les degrés de la hiérarchie sociale ${ }^{51}$.» Cette mise en place sous l'Empire d'instruments statistiques nouveaux, avec la généralisation du passeport que Fouché rend obligatoire, infalsifiable et uniformise, ouvre "des voies nouvelles au contrôle administratif de la sociétés2 ${ }^{2}$, qui se généralisera au XIX ${ }^{e}$ siècle.

Les chapitres consacrés par l'auteur à la police consulaire et impériale sont donc appuyés sur de solides analyses. On pourrait néanmoins souligner que, comme tous ses prédécesseurs, l'auteur place la focale uniquement sur la police politique, malgré un passage rapide sur les problèmes liés à la contrebande aux frontières et aux résistances à la conscription ${ }^{53}$. Son analyse aurait pu être étoffée et parfois nuancée par le recours à une historiographie qui a récemment renouvelé l'approche de la police napoléonienne ${ }^{54}$.

Le dernier apport décisif de l'ouvrage concerne la mise à jour par l'auteur de «l'infinie complexité et l'efficacité des réseaux d'amitié ${ }^{55} »$ de Fouché : pour lui, c'est ce qui explique son parcours, sa montée vers le pouvoir, mais aussi pendant la réaction thermidorienne, le fait qu'il soit relativement peu inquiété. E. de Waresquiel souligne 
en effet pour la première fois l'existence dans l'entourage de Fouché au ministère d'amitiés très durables de celui-ci, soit du temps de l'Oratoire, soit des temps révolutionnaires $^{56}$. Il étoffe également l'analyse des relations de Fouché avec le monde des affaires, notamment les banquiers Ouvrard, Lecomte ou d'Hautmesnil, sous le Directoire, permettant selon l'auteur le coup d'État de fructidor ${ }^{57}$, mais révélant aussi l'aspiration de Fouché à se constituer un patrimoine foncier important.

En décrivant la foule mondaine qui se presse dans les salons de Fouché, E. de Waresquiel insiste pour la première fois sur une autre facette de la personnalité de Fouché, qui n'est plus seulement le solitaire travaillant jour et nuit dans son bureau du ministère, la personnalité détestée, crainte et objet d'effroi que la légende a figée, mais aussi un homme sociable et courtisé, qui entretient ses relations avec ses anciens collègues révolutionnaires comme réussit à se faire aimer des nobles et à se les attacher. Un des aspects les plus novateurs de l'ouvrage réside dans l'analyse de toute la complexité du lien de Fouché au milieu royaliste, étayée de très nombreuses citations de Mémoires de cette noblesse. Dès le début du Consulat, Fouché parvient par des relations privilégiées avec certains nobles de l'Ouest à infiltrer les nombreux réseaux royalistes qui subsistent encore contre Bonaparte ${ }^{58}$. Le ministre entretient également au même moment un rapport très complexe aux émigrés, entre répression et clientélisme, entre soumission à Bonaparte et jeu autonome: alors qu'il voit avec méfiance le retour ans le territoire français de milliers d'émigrés, conscient de la menace qu'ils peuvent représenter pour le régime, il entreprend parallèlement la mise sous surveillance de la masse des émigrés, le sabordage de la commission des émigrés créée par Napoléon afin de contrôler les opérations de radiation, et la constitution, par la distribution de faveurs individuelles, d'une "très solide clientèle parmi l'ancienne noblesse émigrée qui lui vaudra bientôt le soutien d'une partie du faubourg SaintGermain $^{59}$. » Enfin, à partir du début de l'Empire, Fouché est de plus en plus populaire parmi la noblesse parisienne, et certains grands nobles deviennent ses intimes ${ }^{60}$, et continuent à le fréquenter sous la première Restauration.

De fait, la légende noire a occulté la popularité dont a pu jouir Fouché quand il était ministre de la police. E. de Waresquiel cite une dépêche de l'ambassadeur d'Autriche à Paris écrite lors de la disgrâce du ministre en 1810, parlant d'un public : «consterné au dernier point ", et d'un ministre suscitant le "regret général de toute la nation »: « Dans Fouché, [Napoléon] a éloigné le seul de ses ministres qui [...] eût osé mitiger la sévérité de ses ordres, en retarder l'exécution, quelques fois s'y opposer et user de l'influence que lui donnait la supériorité de son esprit pour l'amener à des résolutions plus modérées. [...] Une modération sensible, éprouvée dans chaque occasion, dut naturellement lui ménager une grande popularitéb1.» Enfin, sous les Cent-Jours, Waresquiel restitue bien la complexité du jeu de Fouché, qui "enchaîne les discours hostiles aux Bourbons » officiellement et crée un journal anti-royaliste, tout en aidant ces mêmes royalistes en sous-main ${ }^{62}$, prévenant les plus menacés d'arrestation ou signant des passeports. On est ici loin des jugements de valeurs caricaturaux sur un Fouché sanguinaire, éternel terroriste, haïssant les nobles. Néanmoins, il faudrait nuancer le portrait d'un Fouché populaire que dresse E. de Waresquiel de manière un peu trop générale, cette popularité n'étant à notre sens réelle que dans les milieux nobles parisiens, et ne pouvant être étendue à l'ensemble de la société impériale.

21 Ainsi, la biographie monumentale que livre Emmanuel de Waresquiel est propre à satisfaire un lectorat large, réussissant ainsi son pari. L'amateur passionné y trouvera le 
souffle et les rebondissements d'une vie politique trépidante, l'historien fera la part de la fascination qui ressort parfois au cours de l'ouvrage pour le personnage de Fouché qui définitivement a du mal à s'extraire de sa légende - pour y trouver de nombreuses analyses d'une belle complexité.

\section{NOTES}

1. Victor HUGO, Les travailleurs de la mer, Paris, Gallimard, « Folio classique », 1980, p. 438.

2. Emmanuel de WARESQUIEL, Fouché, les silences de la pieuvre, Paris, Fayard, 2014, p. 24.

3. Louis MADELIN, Joseph Fouché, Paris, Nouveau Monde, 2010 [1901].

4. On peut citer notamment dans cette veine la biographie d'André CASTELOT, Fouché, le double jeu, Paris, Perrin, 1990.

5. Stefan zWEIG, Joseph Fouché, Paris, Grasset, 1969 [1929] : «Je présente donc l'histoire de Joseph Fouché comme une utile et très actuelle contribution à la psychologie de l'homme politique » (p 13). Zweig veut dans cet essai analyser moins les faits que le « caractère, ou plutôt l'absence de caractère du plus parfait des disciples modernes de Machiavel»(p.11). Il dit également avoir voulu livrer la « biographie d'un être absolument amoral » (p. 13).

6. Le chapitre intitulé «les coups de patte de Talleyrand» est extrêmement influencé, jusque dans les tournures de phrases, par quelques pages de Zweig comparant terme à terme Fouché et Talleyrand en en dressant le portrait psychologique et social et en insistant sur leur inimitié réciproque. Voir Stefan ZWEIG, op.cit., p. 148-151.

7. Jean SAVANT, Tel fut Fouché, l'homme qui « gouverna » Bonaparte, Paris, Fasquelle, 1955.

8. Jean TULARD, Joseph Fouché, Paris, Fayard, 1998 : « Ni portrait psychologique à la façon de Zweig ni étude approfondie de la vie de Fouché que rendait inutile la thèse de Madelin, ce livre se veut essentiellement, à travers la forte personnalité de Fouché, une contribution à l'histoire de la police lors de l'époque troublée des années 1793-1815 [...] qui vit s'épanouir la police politique baptisée alors haute police ", p. 15-16. Citons également les biographies de deux historiens anglosaxons également centrées sur le Fouché policier : Hubert COLE, Fouché, the Unprincipled Patriot, Londres, Eyre-Spottiswoode, 1971; Eric A. ARNOLD Jr, Fouché, Napoléon and the General Police, University Press of America, 1979.

9. Emmanuel de WARESQUIEL, op.cit., p. 27-40.

10. Ibid., p. 23.

11. Ancien oratorien, juge puis vice-président du Corps Législatif sous l'Empire grâce à Fouché.

12. Secrétaire particulier de Fouché de 1799 à 1802.

13. Emmanuel de WARESQUIEL, op.cit., p. 17.

14. Ibid., p. 20.

15. Ibid., p. 23.

16. Louis Madelin avait cependant esquissé cette approche, notamment au chapitre XIII où il dresse le portrait psychologique de Fouché et fait l'éloge du père de famille.

17. Emmanuel de Waresquiel, op.cit., p. 48-50.

18. Ibid., p. 382.

19. Ibid., p. 638.

20. Ibid., p. 661.

21. Ibid., p. 64-67. 
22. Ibid., p. 79-81.

23. Ibid., p. 371-373.

24. Ibid., p. 469.

25. Ibid., p. 520-521.

26. Louis MADELIN, op.cit., p. 774.

27. Emmanuel de WARESQUIEL, op.cit., p. 593-594.

28. Meilleure biographie 2014 de Lire magazine, Prix France Télévisions 2015.

29. Emmanuel de WARESQUIEL, op.cit., p. 13.

30. Ibid., p. 34.

31. Ibid., p. 22.

32. Ibid., p. 65. Le passage concerne l'année 1790.

33. Ibid., p. 132.

34. Ibidem.

35. Ibid., p. 187.

36. "Imaginons Fouché au centre d'une toile qui a ses ramifications jusque dans le moindre des quartiers de Paris et dans tous les départements ", Ibid., p. 329.

37. «À force de patauger depuis plus de dix ans dans le cloaque des trahisons, des ambitions et des faux-semblants, il a fini par rentrer tout à fait en lui-même comme une sorte de bernardl'ermite dans sa coquille. Plus que jamais, il cultive une sorte d'indifférence de façade et couvre de son mépris pour les autres les tourments et la nervosité d'un hypersensible », Ibid., p. 386 (le passage concerne l'année 1802).

38. Louis Madelin, op.cit., p. 261. Cet aspect avait déjà été esquissé par Madelin notamment dans son chapitre $\mathrm{X}$ intitulé « la politique du juste milieu », mais a été étoffé par E. de Waresquiel.

39. Emmanuel de Waresquiel, op.cit., p. 411.

40. Ibid., p. 424-425.

41. «De l'opposition sous le gouvernement impérial », annexé aux Mémoires de Gaillard, BnF manuscrits, t VI, NAF 11723 : Fouché y écrit : « Il convient donc de placer sous la protection de [l'Empire] une liberté quelconque de censurer et d'écrire, en conservant dans la main, les moyens d'en mesurer la dose » cité par Emmanuel de Waresquiel, op.cit., p. 414.

42. Ibid., p. 425.

43. Jacques-Olivier BOUDON, préface à Louis Madelin, op.cit.

44. Emmanuel de WARESQUIEL, op.cit., p. 538.

45. Ibid., p. 564-568.

46. Ibid., p. 598.

47. Conservée dans les archives privées de la famille Fouché à Louveciennes.

48. Emmanuel de WARESQUIEL, op.cit., p. 252-254, p. 366 et p. 409-410.

49. Ibid., p. 366.

50. Ibid., p. 162.

51. Ibid., p. 708.

52. Ibid., p. 298.

53. Ibid., p. 423-425.

54. On pense ici notamment à la synthèse opérée par un colloque récent : Jacques-Olivier BOUDON (dir.), Police et gendarmerie dans l'Empire napoléonien, Paris, Editions SPM, 2013.

55. Emmanuel de WARESQUIEL, op.cit., p. 203.

56. On peut citer Pierre-Mathieu Parein placé au sein de la police politique, qui était président de la commission révolutionnaire de Lyon en 1793. Ibid., p. 138.

57. Ibid., p. 220-222.

58. Ibid., p. 286.

59. Ibid., p. 345. 
60. Adrien de Montmorency (futur duc de Laval) et Narbonne (ancien ministre des affaires étrangères de Louis XVI) par exemple.

61. «Rapport du prince de Schwarzenberg. 12 juin 1810 ", cité dans les Mémoires de Clary, p. 313-314.

62. Emmanuel de WARESQUIEL, op.cit., p. 539-540.

\section{AUTEURS}

\section{JEANNE-LAURE LE QUANG}

Institut d'histoire de la Révolution française

Enseignante certifiée en histoire-géographie 